\title{
Synthesis of chiral enantioenriched tetrahydrofuran derivatives
}

\author{
Allan Niidu, ${ }^{a}$ Anne Paju, ${ }^{\text {a }}$ Aleksander-Mati Müürissepp, ${ }^{a}$ Tiiu Kailas, ${ }^{a}$ Tõnis Pehk, ${ }^{b}$ and \\ Margus Lopp ${ }^{\mathbf{a}^{*}}$ \\ ${ }^{a}$ Department of Chemistry, Faculty of Science, Tallinn University of Technology, Tallinn, \\ Estonia \\ ${ }^{b}$ National Institute of Chemical Physics and Biophysics, Tallinn, Estonia
}

E-mail:1opp@chemnet.ee

\begin{abstract}
A simple and short synthetic pathway to novel chiral enantioenriched 2,2-disubstituted tetrahydrofuran derivatives, starting from enantiomeric lactone acids in 2 steps was developed in $36-54 \%$ overall yield. The method enables also to obtain enantioenriched 2,3'-spiro ditetrahydrofuran (1,7-dioxaspiro[4.4]nonane) starting from the spirodilactone ( $R$-1,7dioxaspiro[4.4]nonane-2,6-dione).
\end{abstract}

Keywords: Asymmetric oxidation, chiral tetrahydrofuran derivatives, reduction, 1,7dioxaspiro[4.4]nonane

\section{Introduction}

The tetrahydrofuran structural elements are an essential part in many naturally occurring compounds, like communiols, ${ }^{1}$ acetogenins, ${ }^{2,3}$ polycyclic ethers, ${ }^{4,5,6}$ lignans $^{7}$ etc. Derivatives of 2,2-disubstituted tetrahydrofurans have elicited attention as antitumor agents ${ }^{8}$ and potent VLA-4 antagonists, ${ }^{9,10}$ which could be useful in the treatment of various VLA-4 dependent inflammatory diseases such as asthma, multiple sclerosis and arthritis. 1,7-Dioxaspiro[4.4]nonane (spiroditetrahydrofuran) skeleton exists in the naturally occurring prehispanolones, ${ }^{11}$ leopersins, ${ }^{12}$ syringolides. ${ }^{13}$ Also, this structural element is an essential part in synthetic spironucleosides ${ }^{14}$ and fructose derived molecular scaffolds. ${ }^{15}$

Although, there exist many diastereoselective ${ }^{16-19}$ and enantiospecific ${ }^{16,20}$ methods for synthesizing differently substituted tetrahydrofurans, only a few methods exist to obtain chiral 2,2-disubstituted tetrahydrofuran derivatives. ${ }^{21-24}$ Also, the synthesis of the spiro tetrahydrofuran skeleton, has been realized by many different routes, ${ }^{5,25-27}$ however, only in a few cases has an asymmetric method been used to accomplish this goal. 
In recent years our group has developed a simple and enantioselective method for synthesizing chiral lactone carboxylic acids (Scheme 1) ${ }^{28,29}$ This approach has been applied in the synthesis of 2-alkyl-substituted 2-hydroxyglutaric acid $\gamma$-lactones ${ }^{30}$ homocitric acid ${ }^{31}$, and nucleoside analogues. ${ }^{32,33}$ The easy access and wide possible structural variability of that chiral building block 2 motivated us to broaden the practical scope of the compounds - to use the lactone carboxylic acids for the synthesis of chiral tetrahydrofuran derivatives. In this paper, we report a convenient method for obtaining several novel chiral tetrahydrofurans $\mathbf{3}$ and $\mathbf{7}$ from the corresponding lactone acids $\mathbf{2}$ and spiro ditetrahydrofuran from spirodilactone $\mathbf{2 g}$.<smiles>[R]C1=C(O)C(=O)CC1</smiles>

1
(+)-DET, TBHP, Ti(OiPr) 4

DCM, $-20^{\circ} \mathrm{C}$

$\mathrm{R}=$ alkyl or aryl<smiles>[R][C@]1(C(=O)O)CCC(=O)O1</smiles>

(S)-2

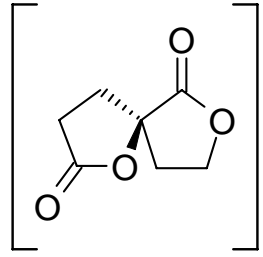

(R)-2g

When $\mathrm{R}=\mathrm{CH}_{2}-\mathrm{CH}_{2}-\mathrm{OH}$

Scheme 1. Asymmetric synthesis of chiral lactone carboxylic acids.

\section{Results and Discussion}

To transform the lactone acid skeleton to the tetrahydrofuran ring we made an attempt to use a direct reduction approach proposed by Verma et al. ${ }^{34}$ for triarylsubstituted dihydrofuranones with neat $\mathrm{BH}_{3} \cdot \mathrm{Me}_{2} \mathrm{~S}(11 \mathrm{eq})$. However, with methylsubstituted lactone 2a this single step procedure at room temperature gave us a two component mixture - hydroxymethyl tetrahydrofuran alcohol $3 \mathbf{a}$ and triol $\mathbf{4 a}$ with $77 \%$ combined isolated yield in a 1:1 ratio (Scheme 2). Also, with benzyloxyethyl lactone acid $2 \mathbf{e}$ the reaction was not selective, resulting in tetrahydrofuran alcohol $3 \mathbf{e}$ and triol $4 \mathbf{e}$ with $90 \%$ overall isolated yield, in a 1:1.4 ratio. Using different borane complexes as reductive agents e.g. $\mathrm{BH}_{3} \cdot \mathrm{NH}_{3}, \mathrm{BH} \cdot \mathrm{THF}, \mathrm{BH}_{3} \cdot \mathrm{Me}_{2} \mathrm{~S} / \mathrm{BF}_{3} \cdot \mathrm{Et}_{2} \mathrm{O}$ did not afford ether 3a from 2a. 


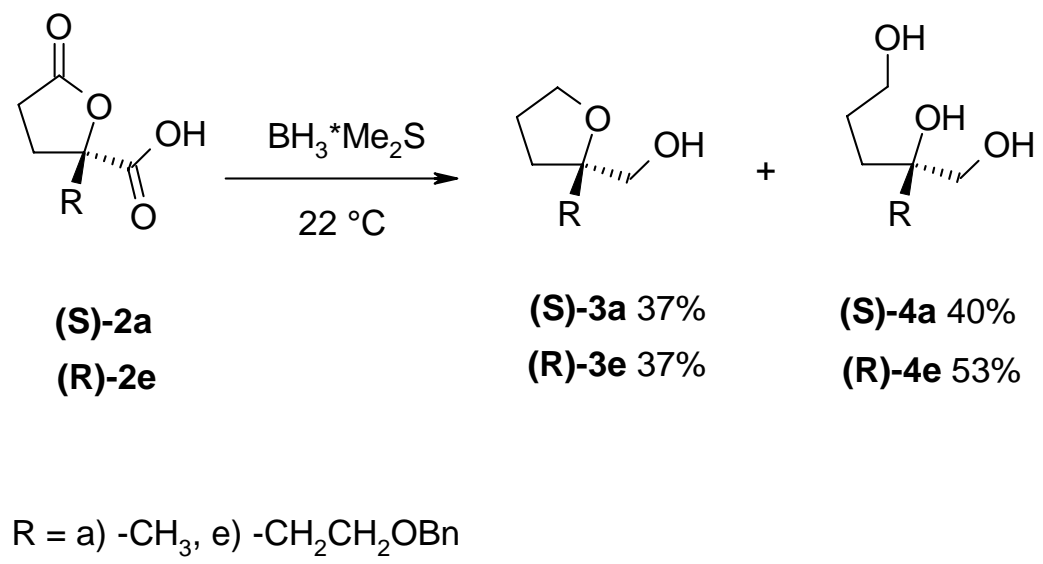

Scheme 2. Direct reduction of carboxylic acids.

To improve the yield of the target tetrahydrofuran derivatives, we applied a two step sequence involving the reduction of lactone carboxylic acid 2 in the first step and reduction of the formed lactone alcohol $\mathbf{5}$ to the ether alcohol $\mathbf{3}$ in the second step. For the reduction of the free carboxylic group we used a protocol proposed by Ravid et al. ${ }^{35}$ For compounds 2a-e the isolated yields of the resulted lactone alcohols 5a-e were in a range of $68-77 \%$ (Scheme 3, Table 1).

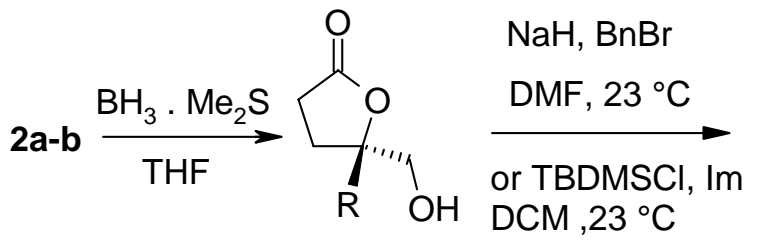

(S)-5a,b<smiles>[R]OC[C@]1([R])CCC(=O)O1</smiles>

1. DIBALH,<smiles>[R]OC[C@]1([R])CCCO1</smiles>

(S)-6a,b,f

(S)-7a,b,f<smiles>[R]C1(CO)CCC(OC[C@]2([R])CCCO2)O1</smiles>

$\mathrm{R}=\mathrm{a}$ ) and f) $-\mathrm{CH}_{3}$, b) $\left.\left.\left.-\mathrm{C}_{2} \mathrm{H}_{5}, \mathrm{c}\right)-\mathrm{Bn}, \mathrm{d}\right)-\mathrm{CH}_{2} \mathrm{OBn}, \mathrm{e}\right)-\mathrm{C}_{2} \mathrm{H}_{4} \mathrm{OBn}$

$\left.\left.\mathrm{R}^{\prime}=\mathrm{a}-\mathrm{b}\right)-\mathrm{Bn}, \mathrm{f}\right)-\mathrm{TBDMS}$

Scheme 3. Synthetic routes to chiral THF-derivatives. 
Table 1. Synthesis of tetrahydrofuran derivatives

\begin{tabular}{ccccc}
\hline Entry & Substrate & $\mathbf{5}$ & $\mathbf{3}$ & $7 \dagger$ \\
\hline $\mathbf{1}$ & 2a & $74 \%$ & & \\
$\mathbf{2}$ & 2b & $73 \%$ & & \\
$\mathbf{3}$ & 2c & $71 \%$ & & \\
$\mathbf{4}$ & $\mathbf{2 d}$ & $68 \%$ & & \\
$\mathbf{5}$ & $\mathbf{2 e}$ & $77 \%$ & & \\
$\mathbf{6}$ & $\mathbf{5 a}$ & & & $48 \%$ \\
$\mathbf{7}$ & $\mathbf{5 b}$ & & & $57 \%$ \\
$\mathbf{8}$ & $\mathbf{5 c}$ & & $75 \%$ & \\
$\mathbf{9}$ & $\mathbf{5 d}$ & & $64 \%$ & \\
$\mathbf{1 0}$ & $\mathbf{5 e}$ & & $70 \%$ & \\
\hline
\end{tabular}

$\dagger$ The yields over two steps (protection and reduction).

There are several options to transform the lactones into cyclic ethers, e.g. $\mathrm{NaBH}_{4} / \mathrm{BF}_{3} \cdot \mathrm{Et}_{2} \mathrm{O},{ }^{36}$ $\mathrm{DIBALH} / \mathrm{Et}_{3} \mathrm{SiH} / \mathrm{BF}_{3} \cdot \mathrm{Et}_{2} \mathrm{O},{ }^{37}$ manganese acetyl complexes $/ \mathrm{PhSiH}_{3}{ }^{38}$ titanocene complexes/ $\mathrm{PMHS} / \mathrm{Et}_{3} \mathrm{SiH} / \mathrm{Amberlyst} 15,{ }^{39}$ and $\mathrm{TiCl}_{4} / \mathrm{TMSOTf}_{\mathrm{Et}} \mathrm{SiH}^{40}{ }^{40}$ ruthenium complexes/ $\mathrm{EtMe}_{2} \mathrm{SiH}^{41}$ The most promising, according to us, is a method, described by Kraus et al. ${ }^{37}$ where DIBALH at $-78^{\circ} \mathrm{C}$ with $\mathrm{Et}_{3} \mathrm{SiH}$ and $\mathrm{BF}_{3} \cdot \mathrm{Et}_{2} \mathrm{O}$ in $\mathrm{DCM}$ is used.

The latter conditions were applied to the starting material 5a, affording in the first attempt a low yield $-<20 \%$ according to GC analysis. The reason may be a low boiling point of the formed product which makes separation of the compound from the reaction mixture complicated when small quantities of starting material $(100 \mathrm{mg})$ are used. Therefore, we protected the hydroxyl group in 5a with a TBDMS protecting group (87-93\% yield) and reduced the protected alcohol with DIBALH/Et ${ }_{3} \mathrm{SiH} / \mathrm{BF}_{3} \cdot \mathrm{Et}_{2} \mathrm{O}$ at $-78{ }^{\circ} \mathrm{C}$ in DCM. The reduction proceeds smoothly, however, under the reaction conditions some cleavage of the TBDMS group occurred in the silane reduction step, giving rise to a mixture of the expected TBDMS ether $\mathbf{7 f}$ together with free alcohol 3a with $77 \%$ combined yield ${ }^{47}$ (Scheme 3 ).

To avoid undesired deprotection of the starting material, we turned to the more stable benzyl protecting group. So, starting materials $\mathbf{5 a}$ and $\mathbf{5 b}$ were protected with benzyl bromide in the presence of an equimolar amount of $\mathrm{NaH}$ in $\mathrm{DMF}$ in $62 \%$ and $73 \%$ yield respectively and were then subjected to reduction with DIBALH/ $\mathrm{Et}_{3} \mathrm{SiH}_{3} \mathrm{BF}_{3} \cdot \mathrm{Et}_{2} \mathrm{O}$ at $-78{ }^{\circ} \mathrm{C}$ in DCM (Scheme 3). As a result, the target tetrahydrofuran derivatives $\mathbf{7 a}$ and $\mathbf{7 b}$ were obtained with $77 \%$ and $78 \%$ yield. The overall yield starting from lactone alcohols $\mathbf{5 a}$ and $\mathbf{5 b}$ were $48 \%$ and $57 \%$ respectively.

The protection/deprotection steps are often complicating the synthetic technologies. Therefore, starting materials 5c-e with an unprotected hydroxyl group were subjected to DIBALH and then $\mathrm{Et}_{3} \mathrm{SiH} / \mathrm{BF}_{3} \cdot \mathrm{Et}_{2} \mathrm{O}$ reduction (Scheme 3). The obtained yield of the reduction step was somewhat lower than in the case of the protected compounds. During the direct Lewis acid promoted silane reduction of 5 intermolecular acetalization of the product 3 with the starting 
compound $\mathbf{5}$ was observed, leading to byproducts 8c-e in 7-10\% yield. This reaction transforms some amount of the starting material to an unreactive acetal and so, diminishes the yield. The byproduct itself is easily separable from the target product by simple chromatography. However, the yields of the target tetrahydrofuran derivatives 3c-e were higher $(64-75 \%)$ than the overall yield of compounds $\mathbf{7 a}$ and $\mathbf{7 b}$ with the corresponding protection steps (48\% and $57 \%$ ) (Table 1$)$. Unmasking to obtain 3a and $\mathbf{3} \mathbf{b}$ would additionally decrease the yield of the whole reaction sequence. So, for the synthesis of compounds 3 the protection-free approach is favourable.

THF derivative 3d is a key intermediate for the synthesis of the bioactive compound (S)-SRI62-834 14 (Scheme 4), an antitumor agent ${ }^{8}$, that has been obtained previously by a multistep sequence that includes enzymatic resolution of the acetylated tetrahydrofuran-2,2-methanol. ${ }^{24}$

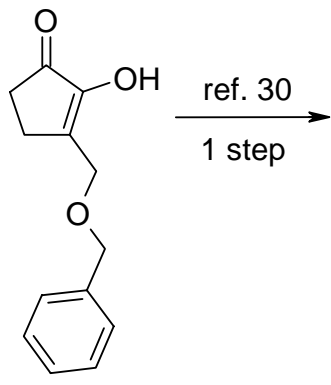

$1 d$

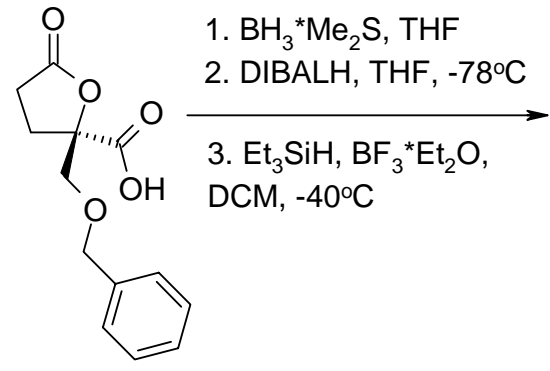

$(R)-2 d$

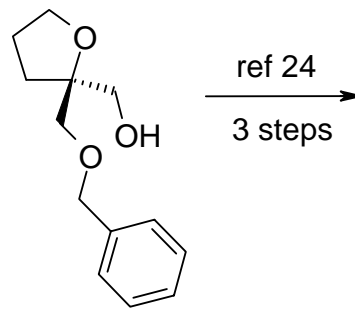

(S)-3d

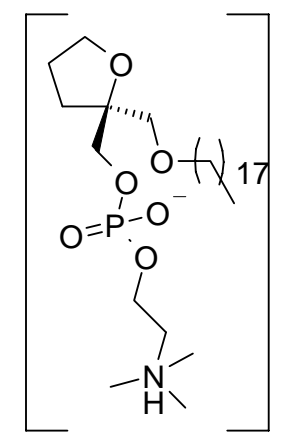

(S)-14

Scheme 4. Formal synthesis of (S)-SRI-62-834 14.

Spirodilactone $\mathbf{2 g}$ was obtained from substituted lactone $\mathbf{2 f}$ by simple lactonisation. ${ }^{42}$ We made an attempt to apply the above described methodology to the reduction of spirodilactone $\mathbf{2 g}$ in order to obtain spiro tetrahydrofuran compound $\mathbf{3 g}$. Thus, we pursued the reduction of $\mathbf{2 g}$ with DIBALH and obtained a mixture of diastereomeric acetal $\mathbf{9}$ and hemiacetal diols $\mathbf{1 0}$ and $\mathbf{1 1}$ as determined by NMR analysis of the crude product (Scheme 5). ${ }^{43}$ These results lead us to search more suitable methods for reducing spirodilactone $\mathbf{2 g}$. A similar over reduction problem was also observed in the synthesis of conformationally restricted spirocyclic nucleosides by Paquette et $a l^{44}$ and they solved the problem by using a low DIBALH concentration in the presence of an excess of Lewis acid (4.5 eq $\left.\mathrm{Me}_{3} \mathrm{SiCl}\right)$. We applied that method to spirodilactone $\mathbf{2 g}$ and obtained lactol 9 together with bicyclic acetal 12 as a single diastereomer (up to $45 \%$ yield; Scheme 5). 


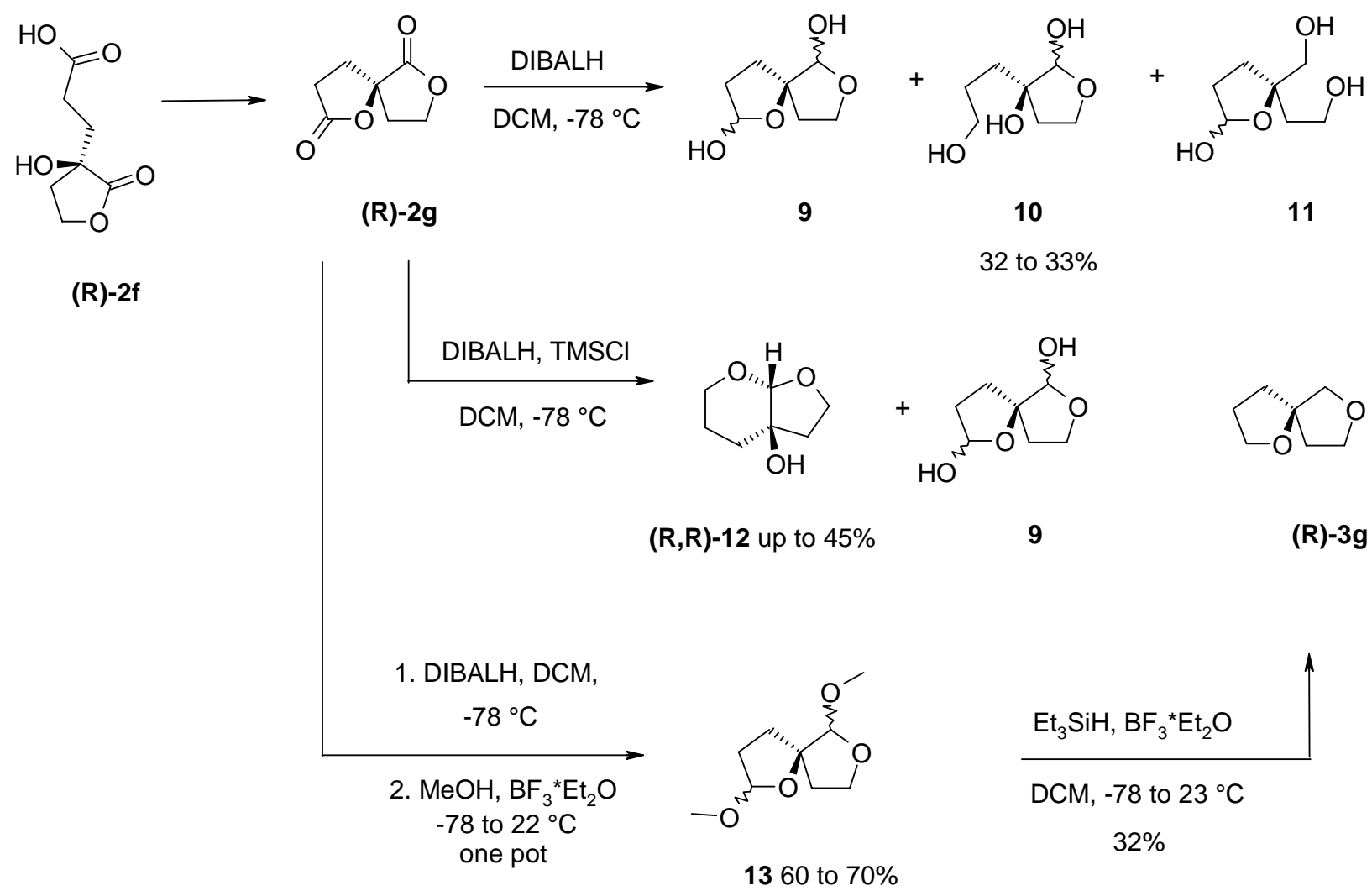

Scheme 5. Synthesis of spirodiether.

Separation of the lactols from the reaction mixture after hydrolysis (containing $\mathrm{Al}(\mathrm{OH})_{3}$ ) appeared to be complicated. In order to improve the yield of acetal 9 (to extract it from the aluminium hydroxide) we used an in situ trapping of the formed lactols as methylacetals $\mathbf{1 3}$ by quenching the DIBALH reaction with an excess of dry $\mathrm{MeOH}$ in the presence of 1 equiv of $\mathrm{BF}_{3} \cdot \mathrm{Et}_{2} \mathrm{O}$. This method afforded stable yields of $13(\sim 70 \%)$. This compound was used in the following step without purification. Silane reduction ${ }^{45,46}$ of $\mathbf{1 3}$ proceeded smoothly (with a modification of the original protocol: a stoichiometric amount of $\mathrm{BF}_{3} \cdot \mathrm{Et}_{2} \mathrm{O}$ was used at $-45^{\circ} \mathrm{C}$ to $\mathrm{rt}$ instead of a catalytic amount at $\mathrm{rt}$ ) to furnish the volatile spiro tetrahydrofuran $3 \mathrm{~g}$ exclusively in $32 \%$ isolated yield after purification (the product partly co-evaporated during solvent removal) (Scheme 5).

In conclusion a short and convenient method for the synthesis of different 2,2-substituted chiral tetrahydrofuran derivatives was developed. The two step sequence gave better overall yield than the direct reduction of the chiral carboxylic acid $-54 \%$ and $37 \%$ respectively. In the case of starting material $\mathbf{2 g}$, the conversion of the hemiacetal $\mathbf{9}$ to the methyl acetal $\mathbf{1 3}$ contributes to the extraction of the latter from the reaction mixture and improves the yield of the reduction step. Subsequent silane reduction gave cleanly the desired compound $3 \mathbf{g}$ with $32 \%$ yield. 


\section{Experimental Section}

General. Chemicals were purchased from Aldrich Chemical Co or Alfa Aesar and were used as received. DCM and DMF were distilled over $\mathrm{CaH}_{2}$ and stored on the $3 \AA$ molecular sieve pellets. THF and ether were distilled over $\mathrm{LiAlH}_{4}$. Precoated silica gel $60 \mathrm{~F} 254$ plates from Merck were used for TLC, whereas for column chromatography silica gel KSK40-100 $\mu \mathrm{m}$ was used. NMR spectra were determined in $\mathrm{CDCl}_{3}$ on Bruker AMX-500 or Bruker Avance USLA 400 spectrometer. Solvent peaks were used as references. 2D FT methods were used for the analysis of synthesized compounds. IR spectra were measured on a Perkin-Elmer Spectrum BX FTIR spectrometer. Mass spectra were recorded on a Hitachi M80B spectrometer using EI (70eV) or a Shimadzu GCMSQP2010 spectrometer using EI $(70 \mathrm{eV})$. Optical rotations were obtained using a Krüss Optronic $\mathrm{GmbH}$ Polarimeter $\mathrm{P}$ 3002. Elemental analyses were performed on a PerkinElmer C, H, N, S-Analyzer 2400. All reactions sensitive to the moisture or oxygen were carried out under Ar atmosphere in oven-dried glassware. Chiral acids 2a-e were synthesized according to previously published methods and each exhibited physical and spectroscopic properties in accordance with data given in literature. ${ }^{34,36}$

Direct reduction of carboxylic acids 2a and 2e. To the carboxylic acid $(1.5 \mathrm{mmol})$ at $-30{ }^{\circ} \mathrm{C}$ was added neat $10 \mathrm{M} \mathrm{BH}_{3} \cdot \mathrm{Me}_{2} \mathrm{~S}(1.5 \mathrm{~mL}, 15 \mathrm{mmol})$ dropwise and the resulting mixture was stirred at $22{ }^{\circ} \mathrm{C}$ for $17 \mathrm{~h}$. Then dry $\mathrm{MeOH}(3.0 \mathrm{~mL})$ was added dropwise at $-20^{\circ} \mathrm{C}$. After stirring at $22{ }^{\circ} \mathrm{C}$ for $1 \mathrm{~h}$ the volatiles were removed and the residue was purified by flash chromatography (petroleum ether/acetone 10:1 to 2:1) to give cyclic ether alcohol along with triol. Tetrahydrofuran 3a: ${ }^{1} \mathrm{H}$ NMR $\left(400 \mathrm{MHz}, \mathrm{CDCl}_{3}\right) \delta 1.19\left(\mathrm{~d}, J=3.1,3 \mathrm{H}, \mathrm{CH}_{3}\right), 1.62$ (ddd, $J$ $=11.2,10.0,6.0,1 \mathrm{H}, \mathrm{H}-3), 1.82-2.01(\mathrm{~m}, 3 \mathrm{H}, \mathrm{H}-3$ and $\mathrm{H}-4), 3.42-3.46\left(\mathrm{~m}, 2 \mathrm{H}, \mathrm{CH}_{2} \mathrm{O}\right), 3.77-$ $3.90(\mathrm{~m}, 2 \mathrm{H}, \mathrm{H}-5),{ }^{13} \mathrm{C}$ NMR $\left(100 \mathrm{MHz}, \mathrm{CDCl}_{3}\right) \delta 23.33\left(\mathrm{CH}_{3}\right), 26.47(\mathrm{C}-4), 33.48(\mathrm{C}-3), 68.07$ (C-5), $68.53\left(\mathrm{CH}_{2} \mathrm{O}\right), 82.95(\mathrm{C}-2)$; triol 4a: ${ }^{1} \mathrm{H}$ NMR (400 MHz, $\left.\mathrm{CDCl}_{3}\right): \delta 1.14$ (s, 3H, $\mathrm{CH}_{3}$ ), $1.52-1.48$ (m, 1H, H-4), 1.54 (dt, $J=7.0,2.1,1 \mathrm{H}, \mathrm{H}-4), 1.69-1.57$ (m, 2H, H-3), $3.38-3.35$ (m, 2H, H-5), $3.59-3.53$ (m, 2H, H-1), $4.90(\mathrm{~s}, 3 \mathrm{H}, \mathrm{OH}) ;{ }^{13} \mathrm{C}$ NMR $\left(100 \mathrm{MHz}, \mathrm{CDCl}_{3}\right): \delta^{13} \mathrm{C}$ NMR (100 MHz, $\left.\mathrm{CDCl}_{3}\right) \delta 23.89\left(\mathrm{CH}_{3}\right), 27.86(\mathrm{C}-4), 35.88(\mathrm{C}-3), 63.71(\mathrm{C}-5), 70.46(\mathrm{C}-1)$, 73.56 (C-2). Tetrahydrofuran 3e: see below. Triol 4e: ${ }^{1} \mathrm{H}$ NMR (500 MHz, DMSO- $\left.D_{6}\right) \delta 1.35$ (m, 2H, H-3), 1.44 (m, 2H, H-4), 3.19 (d, 2H, H-1), 3.35 (m, 2H, H-5), 3.54 (t, 2H, $\left.\mathrm{CH}_{2} \mathrm{O}\right), 4.03$ (s, 1H, OH-2), 4.38 (s, 1H, OH-5), 4.43 (s, 2H, Bn CH 2$), 4.46$ (s, 1H, OH-1), 7.31 (m, 5H, Bn $p$, $o, m) ;{ }^{13} \mathrm{C}$ NMR (DMSO- $\left.D_{6}\right) \delta 26.50(\mathrm{C}-4), 33.38$ (C-3), 36.14 (C-1'), 61.65 (C-5), 66.23 (C-2'), 66.76 (C-1), $71.95\left(\mathrm{Bn} \mathrm{CH}_{2}\right.$ ), 72.43 (C-2), 127.28 (Bn $p$ ), 127.39 (Bn o), 128.20 (Bn $m$ ), 138.72 (Bn $i$ ).

General method for synthesis of lactone alcohols 5a-e. 2-(S)-lactone carboxylic acid $2 \mathbf{2 a}(3 \mathrm{~g}$, $20.8 \mathrm{mmol})$ was dissolved in dry THF $(15 \mathrm{~mL})$ and cooled on ice bath to $4{ }^{\circ} \mathrm{C}$ after which, $\mathrm{BH}_{3} \cdot \mathrm{Me}_{2} \mathrm{~S}(2.37 \mathrm{~mL}, 24.0 \mathrm{mmol})$ was added dropwise over period of $20 \mathrm{~min}$. The resulting mixture was stirred at $23{ }^{\circ} \mathrm{C}$ for $1.5 \mathrm{~h}$ to $2.0 \mathrm{~h}$ (endpoint was confirmed by TLC). Then $\mathrm{MeOH}$ $(1.25 \mathrm{~mL})$ was carefully added to destroy borane complex. The solvents were removed in vacuo, then $\mathrm{MeOH}(1.25 \mathrm{~mL})$ was added and the volatiles were removed - the procedure was repeated 
once to yield the crude product as light yellow viscous oil. Purification was achieved by flash chromatography $\left(\mathrm{SiO}_{2}\right.$, petroleum ether /EtOAc 3:1 to 1:1).

(S)-5-Hydroxymethyl-5-methyl-dihydro-furan-2-one 5a. The title compound was synthesized in $20.8 \mathrm{mmol}$ scale and recrystallization from petroleum ether/ $\mathrm{Et}_{2} \mathrm{O}$ obtained as white solid (2.01 g, $15.5 \mathrm{mmol}, 74 \%)$. $[\alpha]_{\mathrm{D}}{ }^{23}=+12.7$ (c 3.47, AcOEt); ${ }^{1} \mathrm{H} \mathrm{NMR}\left(500 \mathrm{MHz}, \mathrm{CDCl}_{3}\right): \delta 1.36(\mathrm{~s}, 3 \mathrm{H}$, $\mathrm{CH}_{3}$ ), 1.92 (ddd, 1H, H-4), 2.36 (ddd, 1H, H-4), 2.58 (ddd, 1H, H-3), 2.69 (ddd, 1H, H-3), 2.73 (bs, $1 \mathrm{H}, \mathrm{OH}) 3.51$ (d, 1H, CH2-OH), 3.70 (d, 1H, CH2-OH); $\left.{ }^{13} \mathrm{C} \mathrm{NMR} \mathrm{(125} \mathrm{MHz,} \mathrm{CDCl}\right): \delta$ $23.01\left(\mathrm{CH}_{3}\right), 29.54(\mathrm{C}-4), 29.57(\mathrm{C}-3), 68.31(\mathrm{C}-\mathrm{OH}), 86.66(\mathrm{C}-5), 177.38$ (C-2); IR (KBr) 3412, 2978, 2938, 1759, 1649, 1459, 1419, 1384, 1304, 1213, 1162, 1130, 1099, 1061, 1011, 945; MS $(\mathrm{m} / \mathrm{z}): 131(\mathrm{M}+1)^{+}, 115,99$ (base) $, 71,56$.

(S)-5-Ethyl-5-hydroxymethyldihydrofuran-2-one $5 \mathbf{b}$. The title compound was synthesized in $0.7 \mathrm{mmol}$ scale in good yield to obtain a colorless oil $(73 \mathrm{mg}, 73 \%)$, which solidified upon smearing with glass stick. $\left.[\alpha]_{\mathrm{D}}{ }^{25}=+11.5(c) 3.29, \mathrm{CHCl}_{3}\right) ;{ }^{1} \mathrm{H} \mathrm{NMR}\left(500 \mathrm{MHz}, \mathrm{CDCl}_{3}\right): \delta 0.94(\mathrm{t}$, $\left.J=2 \times 7.5 \mathrm{~Hz}, 3 \mathrm{H}, \mathrm{CH}_{3}\right), 1.69\left(\mathrm{dt}, J=3 \times 7.5,14.2,1 \mathrm{H}, \mathrm{CH}_{2}\right), 1.71\left(\mathrm{dt}, J=3 \times 7.5,14.2,1 \mathrm{H}, \mathrm{CH}_{2}\right)$, 1.98 (ddd, $J=7.3,10.7,13.0 \mathrm{~Hz}, 1 \mathrm{H}, \mathrm{H}-4), 2.26$ (ddd, $J=5.8,10.8,13.0 \mathrm{~Hz}, 1 \mathrm{H}, \mathrm{H}-4), 2.55$ (ddd, $J=5.8,10.7,18.2 \mathrm{~Hz}, 1 \mathrm{H}, \mathrm{H}-3), 2.70$ (ddd, $J=7.3,10.8,18.2 \mathrm{~Hz}, 1 \mathrm{H}, \mathrm{H}-4), 3.01$ (bs, 1H, OH), $3.54\left(\mathrm{~d}, J=12.1 \mathrm{~Hz}, 1 \mathrm{H}, \mathrm{CH}_{2} \mathrm{OH}\right), 3.73\left(\mathrm{~d}, J=12.1 \mathrm{~Hz}, 1 \mathrm{H}, \mathrm{CH}_{2} \mathrm{OH}\right) ;{ }^{13} \mathrm{C} \mathrm{NMR}(125 \mathrm{MHz}$, $\left.\mathrm{CDCl}_{3}\right): \delta 7.42\left(\mathrm{Et} \mathrm{CH}_{3}\right), 26.87(\mathrm{C}-4), 28.97\left(\mathrm{Et} \mathrm{CH}_{2}\right), 29.62(\mathrm{C}-3), 66.93\left(\mathrm{CH}_{2} \mathrm{OH}\right), 89.39(\mathrm{C}-5)$, 177.90 (C-2); IR (KBr) 3433(OH), 2974, 2944, 2886, 1766(C=O), 1464, 1418, 1331, 1219 , 1160, 1120, 1069, 976, 936; MS m/z: $145(\mathrm{M}+1)^{+}, 127,113$ (base), 98, 95, 71, 57, 55, 41; anal. calcd. for $\mathrm{C}_{7} \mathrm{H}_{12} \mathrm{O}_{3}$ : C, 58.32; H, 8.39. Found C, 57.95; H, 8.49.

(R)-5-Benzyl-5-hydroxymethyldihydrofuran-2-one 5c. The title compound was synthesized in $0.45 \mathrm{mmol}$ scale to yield the title compound as white solid $(67 \mathrm{mg}, 0.33 \mathrm{mmol}, 71 \%) .[\alpha]_{\mathrm{D}}{ }^{23}=$ $+56.6\left(\right.$ c 6.58, $\left.\mathrm{CHCl}_{3}\right) ;{ }^{1} \mathrm{H} \mathrm{NMR}\left(400 \mathrm{MHz}, \mathrm{CDCl}_{3}\right) \delta 1.96(\mathrm{~m}, 1 \mathrm{H}, \mathrm{H}-3), 2.07$ (m, 1H, H-4), 2.21 (ddd, $J=7.2,10.4,12.5 \mathrm{~Hz}, 1 \mathrm{H}, \mathrm{H}-4), 2.45$ (ddd, $J=5.1,10.4,17.5 \mathrm{~Hz}, 1 \mathrm{H}, \mathrm{H}-3), 2.85$ (d, $J=14.0$ $\mathrm{Hz}, 1 \mathrm{H}, \mathrm{Bn} \mathrm{CH}_{2}$ ), 3.06 (d, $J=14.0 \mathrm{~Hz}, 1 \mathrm{H}, \mathrm{Bn} \mathrm{CH}_{2}$ ), 3.60 (d, $\left.J=12.1 \mathrm{~Hz}, 1 \mathrm{H}, \mathrm{CH}_{2} \mathrm{OH}\right), 3.76$ (d, $\left.J=11.9 \mathrm{~Hz}, 1 \mathrm{H}, \mathrm{CH}_{2} \mathrm{OH}\right), 7.29(\mathrm{~m}, 5 \mathrm{H}, \mathrm{Bn} m, p, o) ;{ }^{13} \mathrm{C} \mathrm{NMR}\left(100 \mathrm{MHz}, \mathrm{CDCl}_{3}\right): \delta 26.63(\mathrm{C}-4)$, $29.25(\mathrm{C}-3), 41.97\left(\mathrm{Bn} \mathrm{CH}_{2}\right), 67.63\left(\mathrm{CH}_{2} \mathrm{OH}\right), 88.19(\mathrm{C}-5), 127.21(\mathrm{Bn} p), 128.64(\mathrm{Bn} m)$, 130.43 (Bn $o$ ), 134.76 (Bn i), 177.15 (C-2); IR (film) 3434(OH), 2929, 1767(C=O), 1496, 1456, 1416, 1187, 1061, 942, 705; MS m/z: $206(\mathrm{M})^{+}, 188,175,129,115$ (base), 91, 77, 65, 55, 41; HRMS calcd. for (M) ${ }^{+} \mathrm{C}_{12} \mathrm{H}_{14} \mathrm{O}_{3}$ : 206.0942; found: 206.0944.

(S)-5-Benzyloxymethyl-5-hydroxymethyldihydro-furan-2-one $5 \mathbf{d}$. The title compound wa synthesized in $0.46 \mathrm{mmol}$ scale to yield the title compound as light yellow liquid (74 $\mathrm{mg}, 0.31$ mmol, 68\%). $[\alpha]_{\mathrm{D}}{ }^{23}=+8.1\left(\right.$ c 3.29, $\left.\mathrm{CHCl}_{3}\right) ;{ }^{1} \mathrm{H}$ NMR $\left(400 \mathrm{MHz}, \mathrm{CDCl}_{3}\right): \delta 2.14$ (m, 2H, H-4), $2.61(\mathrm{~m}, 2 \mathrm{H}, \mathrm{H}-3), 3.55$ (dd, $J=10.2,21.9 \mathrm{~Hz}, 2 \mathrm{H}, \mathrm{CH}_{2} \mathrm{O}$ ), 3.70 (dd, J=12.0, $53.0 \mathrm{~Hz}, 2 \mathrm{H}$, $\left.\mathrm{CH}_{2} \mathrm{OH}\right), 4.53(\mathrm{~s}, 2 \mathrm{H}, \mathrm{Bn} \mathrm{CH}), 7.31$ (m, 5H, Bn $\left.m, p, o\right) ;{ }^{13} \mathrm{C}$ NMR (100 MHz, $\left.\mathrm{CDCl}_{3}\right): \delta 25.82$ (C-4), $29.31(\mathrm{C}-3), 65.62\left(\mathrm{CH}_{2} \mathrm{OH}\right), 72.55\left(\mathrm{CH}_{2} \mathrm{O}\right), 73.84\left(\mathrm{Bn} \mathrm{CH}_{2} \mathrm{O}\right), 87.67(\mathrm{C}-5), 127.74(\mathrm{Bn}$ $o$ ), 128.01 ( $\mathrm{Bn} p$ ), 128.62 (Bn $m$ ), 137.63 (Bn i), 177.47 (C-2); IR (neat) 3020, 2400, 1773, 1216, 752, 669; MS m/z: $205(\mathrm{M}-31)^{+}, 130,115,91,55,41$; HRMS calcd. for $(\mathrm{M}-106)^{+} \mathrm{C}_{6} \mathrm{H}_{10} \mathrm{O}_{3}$ : 130.0630, found: 130.0635 . 
(R)-5-Benzyloxyethyl-5-hydroxymethyl-dihydrofuran-2-one 5e. The tilte compound was synthesized in $0.18 \mathrm{mmol}$ scale yielded title compound as light yellow syrup ( $35 \mathrm{mg}, 0.14 \mathrm{mmol}$, $77 \%) \cdot[\alpha]_{\mathrm{D}}{ }^{24}=-7.0\left(c 7.63, \mathrm{CHCl}_{3}\right) ;{ }^{1} \mathrm{H} \mathrm{NMR}\left(400 \mathrm{MHz}, \mathrm{CDCl}_{3}\right): \delta 2.01\left(\mathrm{~m}, 2 \mathrm{H}, \mathrm{Et} \mathrm{CH}_{2}\right), 2.06$ (m, 1H, H-4), 2.27 (m, 1H, H-4), $2.56(\mathrm{~m}, 2 \mathrm{H}, \mathrm{H}-3), 3.58\left(\mathrm{~m}, 2 \mathrm{H}\right.$, Et $\left.\mathrm{CH}_{2} \mathrm{O}\right), 3.60(\mathrm{~s}, 2 \mathrm{H}$, $\left.\mathrm{CH}_{2} \mathrm{OH}\right), 3,65\left(\mathrm{~m}, 1 \mathrm{H}, \mathrm{Et} \mathrm{CH}_{2} \mathrm{O}\right), 4.48$ (s, Bn $\left.\mathrm{CH}_{2} \mathrm{O}\right), 7.30(\mathrm{~m}, 5 \mathrm{H}, \mathrm{Bn}) ;{ }^{13} \mathrm{C} \mathrm{NMR}(100 \mathrm{MHz}$, $\left.\mathrm{CDCl}_{3}\right): \delta 28.97(\mathrm{C}-3), 29.03(\mathrm{C}-4), 36.91(\mathrm{Et} \mathrm{CH}), 65.77\left(\mathrm{Et} \mathrm{CH}_{2} \mathrm{O}\right), 66.85\left(\mathrm{CH}_{2} \mathrm{OH}\right), 73.73$ $\left(\mathrm{Bn} \mathrm{CH}_{2} \mathrm{O}\right.$ ), 87.63 (C-5), 128.03 (Bn $o$ ), 128.19 (Bn $p$ ), 128.77 (Bn $m$ ), 137.63 (Bn $\left.i\right), 177.06$ (C2); IR (neat) 3444, 2928, 2871, 1769, 1208, 1097; MS m/z: 219 (M-31) ${ }^{+}, 172,159$ (M-91), 126, 107, 91 (Base), 79, 65, 44; HRMS: calcd. for (M-91) ${ }^{+} \mathrm{C}_{7} \mathrm{H}_{11} \mathrm{O}_{4}$ : 159.0656; found: 159.0653 .

Benzylation of $5 \mathbf{a}$ and $5 \mathbf{b}$. To the lactone $5 \mathbf{a}(100 \mathrm{mg}, 0.77 \mathrm{mmol})$ dissolved in dry DMF (1.5 $\mathrm{mL})$, sodium hydride $(27 \mathrm{mg}, 1.15 \mathrm{mmol})$ and $\mathrm{BnBr}(165 \mathrm{uL}, 1.39 \mathrm{mmol})$ were added at $0{ }^{\circ} \mathrm{C}$. The resulting solution was stirred at $23{ }^{\circ} \mathrm{C}$ for $48 \mathrm{~h}$, after which water $(0.5 \mathrm{~mL})$ was added dropwise on ice bath to quench the reaction. Then DCM $(2.5 \mathrm{~mL})$ was added and the layers separated. Water phase was extracted with DCM $(2 \times 2.5 \mathrm{~mL})$ and the organics washed with brine $(1.0 \mathrm{~mL})$, then dried over $\mathrm{MgSO}_{4}$, filtered and concentrated to yield crude product, which was purified by flash chromatography $\left(\mathrm{SiO}_{2}\right.$, petroleum ether /EtOAc 3:1 to 1:1) giving compound (105 mg, 62\%) as a colorless oil.

(S)-5-Benzyloxymethyl-5-methyldihydrofuran-2-one 6a. Synthesis in $0.77 \mathrm{mmol}$ scale gave the title compound $(105 \mathrm{mg}, 62 \%)$ as a colorless oil. ${ }^{1} \mathrm{H}$ NMR $\left(500 \mathrm{MHz}, \mathrm{CDCl}_{3}\right): \delta 1.39$ (s, $3 \mathrm{H}$, $\mathrm{CH}_{3}$ ), 1.94 (ddd, $J=8.4,10.3,12.8 \mathrm{~Hz}, 1 \mathrm{H}, \mathrm{H}-4$ ), 2.35 (ddd, $J=4.8,10.4,12.8 \mathrm{~Hz}, 1 \mathrm{H}, \mathrm{H}-4$ ), 2.54 (ddd, $J=4.8,10.4,17.9 \mathrm{~Hz}, 1 \mathrm{H}, \mathrm{H}-3), 2.74$ (ddd, $J=8.4,10.4,17.9 \mathrm{~Hz}, 1 \mathrm{H}, \mathrm{H}-3), 3.44$ (d, $J=10.1$ $\mathrm{Hz}, 1 \mathrm{H}, \mathrm{CH}_{2} \mathrm{O}$ ), 3.52 (d, J=10.1 Hz, 1H, $\mathrm{CH}_{2} \mathrm{O}$ ), 4.53 (d, J=12.1 Hz, 1H, Bn $\mathrm{CH}_{2}$ ), 4.59 (d, $J=12.1 \mathrm{~Hz}, 1 \mathrm{H}, \mathrm{Bn} \mathrm{CH} 2), 7.31(\mathrm{~m}, 3 \mathrm{H}, p, o), 7.36(\mathrm{~m}, 2 \mathrm{H}, m) ;{ }^{13} \mathrm{C} \mathrm{NMR}\left(125 \mathrm{MHz}, \mathrm{CDCl}_{3}\right): \delta$ $23.74\left(\mathrm{CH}_{3}\right), 29.68(\mathrm{C}-3), 30.73(\mathrm{C}-4), 73.52\left(\mathrm{Bn} \mathrm{CH}_{2}\right), 75.82\left(\mathrm{CH}_{2} \mathrm{O}\right), 85.30(\mathrm{C}-5), 127.48$ (oBn), 127.73 ( $p$-Bn), 128.41 ( $m$-Bn), 137.68 ( $i$-Bn), 177.13 (C-2); IR (neat) 4060, 3520, 3089, 3064, 3032, 2977, 2936, 2867, 1958, 1772, 1604, 1497, 1454, 1416, 1381, 1367,1286, 1231, 1212, 1158, 1099, 1028, 1011, 943; MS m/z: $220(\mathrm{M})^{+}, 114,99$ (base), 91, 71, 65, 55, 43; anal. calcd. for $\mathrm{C}_{13} \mathrm{H}_{16} \mathrm{O}_{3}$ : C, 70.89; H, 7.32; found: C, 70.41; H, 7.29.

(S)-5-Benzyloxymethyl-5-ethyldihydrofuran-2-one 6b. Synthesis in $0.48 \mathrm{mmol}$ scale gave benzyl ether (82 mg, $0.35 \mathrm{mmol}, 73 \%$ ) as colorless oil. ${ }^{1} \mathrm{H} \mathrm{NMR}\left(400 \mathrm{MHz}, \mathrm{CDCl}_{3}\right): \delta 0.89$ (t, $\left.J=7.5,3 \mathrm{H}, \mathrm{CH}_{3}\right), 1.66$ (m, 2H, $\mathrm{CH}_{2}$ ), 1.93 (ddd, $\left.J=8.5,10.6,12.9,1 \mathrm{H}, \mathrm{H}-4\right), 2.20$ (ddd, $J=4.5$, 10.6, 12.9, 1H, H-4), 2.44 (ddd, $J=4.5,10.6,15.1,1 \mathrm{H}, \mathrm{H}-3$ ), 2.67 (ddd, $J=8.5,10.6,18$., $1 \mathrm{H}, \mathrm{H}-$ 3), 3.46 (q, $\left.J=10.1,2 \mathrm{H}, \mathrm{CH}_{2} \mathrm{O}\right), 4.50$ (q, $\left.J=12.0,2 \mathrm{H}, \mathrm{Bn} \mathrm{CH}_{2}\right), 7.27(\mathrm{~m}, 5 \mathrm{H}, \mathrm{Bn} o, p, m) ;{ }^{13} \mathrm{C}$ NMR (100 MHz, $\left.\mathrm{CDCl}_{3}\right)$ : d $7.62\left(5-\mathrm{CH}_{3}\right), 28.37$ (C-4), $29.78\left(5-\mathrm{CH}_{2}\right), 29.84$ (C-3), 73.75 (5$\left.\mathrm{CH}_{2} \mathrm{O}\right), 74.73\left(\mathrm{Bn}_{-} \mathrm{CH}_{2}\right), 87.98(\mathrm{C}-5), 127.65(o), 127.87(p), 128.58(m), 137.93(i), 177.50(\mathrm{C}-$ 2); IR 3030, 2972, 2926, 1770, 1496, 1454, 1416, 1366, 1221, 1158, 1100, 940, 738, 699; MS m/z: $205(\mathrm{M}-29)^{+}, 179,159,143,128,113$ (base), 107, 91, 71, 57; anal. calcd. for $\mathrm{C}_{14} \mathrm{H}_{18} \mathrm{O}_{3}$ : C, 71.77; H, 7.74; found: C, 72.16; H, 7.72.

General method for synthesis of tetrahydrofurans 7a-b and 3c-e. Lactone benzyl ether 6a $(0.27 \mathrm{mmol})$ was dissolved in DCM $(1.0 \mathrm{~mL})$ and cooled to $-78^{\circ} \mathrm{C}$, then DIBALH was added 
dropwise and the resulting solution stirred for $3 \mathrm{~h}$. Reaction mixture was quenched with water $(200 \mathrm{uL})$ and the temperature was allowed to reach $0{ }^{\circ} \mathrm{C}$, then DCM $(2.5 \mathrm{~mL})$ was added. The resulting mixture was stirred at $22{ }^{\circ} \mathrm{C}$ for $1 \mathrm{~h}$, filtered through Celite and the solids were washed with DCM $(3 \times 2.5 \mathrm{~mL})$. TLC showed presence of one product. After removal of solvents, the residue was dissolved in $\mathrm{DCM}(1.0 \mathrm{~mL})$ and $\mathrm{Et}_{3} \mathrm{SiH}(70 \mathrm{uL}, 0.41 \mathrm{mmol})$ was added. Then the reaction mixture was cooled to $-45^{\circ} \mathrm{C}$ and $\mathrm{BF}_{3} \cdot \mathrm{Et}_{2} \mathrm{O}(40 \mathrm{uL}, 0.31 \mathrm{mmol})$ was added dropwise. After stirring for $3 \mathrm{~h}$, the reaction was quenched with aq. $\mathrm{NaHCO}_{3}$ solution $(10 \%, 0.5 \mathrm{~mL})$. The layers were separated and the water phase was extracted with DCM $(3 \times 2.5 \mathrm{~mL})$. The combined organics were dried over $\mathrm{MgSO}_{4}$ and concentrated to give crude product. Further purification was achieved by flash chromatography (petroleum ether /EtOAc 5:1 to 1:1).

(2S)-2-[(Benzyloxy)methyl]-2-methyltetrahydro-furan 7a Obtained as a colorless liquid (43 mg, 77\%). ${ }^{1} \mathrm{H}$ NMR (400 MHz, $\left.\mathrm{CDCl}_{3}\right): \delta 1.23\left(\mathrm{~d}, J=6.6,3 \mathrm{H}, \mathrm{CH}_{3}\right), 1.60(\mathrm{~m}, 1 \mathrm{H}, \mathrm{H}-1), 1.76(\mathrm{~m}$, $3 \mathrm{H}, \mathrm{H}-1, \mathrm{H}-5), 3.33$ (dd, $J=9.71,2 \mathrm{H}, \mathrm{CH}_{2} \mathrm{O}$ ), 3.84 (m, 2H, H-4), 4.56 (dd, $2 \mathrm{H}, J=12.35,13.45$, $\left.\mathrm{Bn} \mathrm{CH}_{2} \mathrm{O}\right), 7.28$ (m, 5H, Bn $\left.o, p, m\right) ;{ }^{13} \mathrm{C}$ NMR $\left(100 \mathrm{MHz}, \mathrm{CDCl}_{3}\right): \delta 24.32\left(\mathrm{CH}_{3}\right), 26.37(\mathrm{C}-5)$, 34.63 (C-3), 68.19 (C-5), $73.62\left(\mathrm{Bn} \mathrm{CH}_{2}\right), 76.53\left(\mathrm{CH}_{2} \mathrm{O}\right), 82.40(\mathrm{C}-2), 127.61(p-\mathrm{Bn}), 127.68(o-$ $\mathrm{Bn}), 128.48$ ( $m$-Bn), 138.84 (i-Bn); IR (neat) 3088, 3064, 3030, 2970, 2866, 1810, 1604, 1497, 1454, 1370, 1310, 1272, 1206, 1103, 1050, 736, 698, 606; MS (m/z): $206(\mathrm{M})^{+}, 191,175,148$, $135,119,107,91,85$ (base), 77, 65, 43; anal. calcd. for $\mathrm{C}_{13} \mathrm{H}_{18} \mathrm{O}_{2}$ : C, 75.69; H, 8.80; found: $\mathrm{C}$, $75.56 ; \mathrm{H}, 8.78$.

(S)-5-Benzyloxymethyl-5-ethyltetrahydrofuran $7 \mathbf{b}$. Synthesis in $0.26 \mathrm{mmol}$ scale yielded the title compound as a colorless liquid (45 mg, 78\%). ${ }^{1} \mathrm{H}$ NMR (400 MHz, $\left.\mathrm{CDCl}_{3}\right): \delta 0.90(\mathrm{~m}, 3 \mathrm{H}$, Et $\left.\mathrm{CH}_{3}\right), 1.63\left(\mathrm{~m}, 3 \mathrm{H}, \mathrm{H}-3\right.$ and $\mathrm{Et}_{\mathrm{CH}}$ ) $, 1.86(\mathrm{~m}, 3 \mathrm{H}, \mathrm{H}-3$ and $\mathrm{H}-4), 3.33$ (q, $\left.J=9.5,2 \mathrm{H}, \mathrm{CH}_{2} \mathrm{O}\right)$, 3.82 (q, $J=6.1,2 \mathrm{H}, \mathrm{H}-5), 4.55$ (m, 2H, Bn $\left.\mathrm{CH}_{2}\right), 7.27$ (m, 5H, Bn $\left.o, p, m\right) ;{ }^{13} \mathrm{C}$ NMR $(100 \mathrm{MHz}$, $\left.\mathrm{CDCl}_{3}\right): \delta 8.53\left(\mathrm{Et} \mathrm{CH}_{3}\right), 26.53(\mathrm{C}-4), 29.73\left(\mathrm{Et} \mathrm{CH}_{2}\right), 32.37$ (C-3), $68.37(\mathrm{C}-5), 73.59\left(\mathrm{Bn} \mathrm{CH}_{2}\right)$, $74.55\left(\mathrm{CH}_{2} \mathrm{O}\right), 84.82(\mathrm{C}-2), 127.58(p-\mathrm{Bn}), 127.67(o-\mathrm{Bn}), 128.43(m-\mathrm{Bn}), 138.83(i-\mathrm{Bn}) ; \mathrm{IR}$ (film): 3440, 3085, 3061, 3028, 2949, 2868, 1950, 1880, 1813, 1758, 1604, 1583, 1496, 1454, 1400, 1330, 1296, 1200, 1147, 1123, 1087, 1039, 957, 702; MS (m/z): $191(\mathrm{M}-29)^{+}, 161,149$, 114, 99 (base), 91, 57; anal. calcd. for $\mathrm{C}_{14} \mathrm{H}_{20} \mathrm{O}_{2}: \mathrm{C}, 76.33 ; \mathrm{H}, 9.15$; found: C, 75.98; H, 9.21.

((R)-2-Benzyltetrahydrofuran-2-yl)-methanol 3c. Synthesis in $0.46 \mathrm{mmol}$ scale yielded the title compound as a colorless liquid (66 mg, 75\%). $[\alpha]_{\mathrm{D}}{ }^{23}=-1.5\left(c\right.$ 12.76, $\left.\mathrm{CHCl}_{3}\right) ;{ }^{1} \mathrm{H}$ NMR (400 $\left.\mathrm{MHz}, \mathrm{CDCl}_{3}\right): \delta 1.67$ (m, 2H, H-3, H-4), 1.83 (m, 2H, H-3, H-4), 2.13 (bs, $\left.1 \mathrm{H}, \mathrm{OH}\right), 2.80(\mathrm{~m}$, $\left.2 \mathrm{H}, \mathrm{Bn} \mathrm{CH}_{2}\right), 3.49\left(\mathrm{~m}, 2 \mathrm{H}, \mathrm{CH}_{2} \mathrm{OH}\right), 3.79(\mathrm{~m}, 2 \mathrm{H}, \mathrm{H}-5), 7.28(\mathrm{~m}, 5 \mathrm{H}, \mathrm{Bn} o, p, m) ;{ }^{13} \mathrm{C} \mathrm{NMR}$ (100 MHz, $\left.\mathrm{CDCl}_{3}\right): \delta 26.53(\mathrm{C}-4), 31.35(\mathrm{C}-3), 42.38\left(\mathrm{Bn} \mathrm{CH}_{2}\right), 67.35\left(\mathrm{CH}_{2} \mathrm{OH}\right), 68.63(\mathrm{C}-5)$, 85.69 (C-2), 126.47 (p-Bn), $128.39(o-\mathrm{Bn}), 130.75(m-\mathrm{Bn}), 137.82(s-\mathrm{Bn})$; IR $\left(\mathrm{CHCl}_{3}\right)$ : 3440, 3085, 3061, 3028, 2949, 2868, 1950, 1880, 1813, 1758, 1604, 1583, 1496, 1454, 1400, 1330, 1296, 1200, 1147, 1123, 1087, 1039, 957, 702; MS (m/z): $192(\mathrm{M})^{+}, 161,128,115,101$ (base), 91, 83, 65; HRMS calcd. for (M) ${ }^{+} \mathrm{C}_{12} \mathrm{H}_{16} \mathrm{O}_{2}$ : 192.1149; found 192.1156; anal. calcd. for $\mathrm{C}_{12} \mathrm{H}_{16} \mathrm{O}_{2}$ : C, 74.97; $\mathrm{H}, 8.39$; found: $\mathrm{C}, 74.72 ; \mathrm{H}, 8.44$.

((S)-2-Benzyloxymethyltetrahydrofuran-2-yl)-methanol 3d. Synthesis in $0.19 \mathrm{mmol}$ scale yielded the title compound as a colorless liquid $(23 \mathrm{mg}, 64 \%) .{ }^{1} \mathrm{H}$ NMR $\left(400 \mathrm{MHz}, \mathrm{CDCl}_{3}\right): \delta$ 
$1.85(\mathrm{~m}, 4 \mathrm{H}, \mathrm{H}-3$ and $\mathrm{H}-4), 2.10(\mathrm{~s}, 1 \mathrm{H}, \mathrm{OH}), 3.43$ (dd, J=9.4, 39.4, 2H, CH $2 \mathrm{O}$ ), 3.56 (dd, $\left.J=11.3,37.0,2 \mathrm{H}, \mathrm{CH}_{2} \mathrm{OH}\right), 3.84$ (t, $\left.J=6.4,2 \mathrm{H}, \mathrm{H}-5\right), 4.54$ (q, $J=12.2,2 \mathrm{H}, \mathrm{Bn} \mathrm{CH}_{2} \mathrm{O}$ ), 7.30 (m, $5 \mathrm{H}, \mathrm{Bn} o, p, m) ;{ }^{13} \mathrm{C}$ NMR (100 MHz, $\left.\mathrm{CDCl}_{3}\right): \delta 26.38(\mathrm{C}-4), 30.67$ (C-3), $66.43(\mathrm{C}-5), 68.86$ $\left(\mathrm{CH}_{2} \mathrm{OH}\right), 73.43\left(\mathrm{CH}_{2} \mathrm{O}\right), 73.80\left(\mathrm{Bn} \mathrm{CH}_{2} \mathrm{O}\right), 84.39$ (C-2), 127.80 (o-Bn), 127.85 (p-Bn), 128.60 (m-Bn), 138.38 (s-Bn); IR (neat): 3439, 3089, 3064, 3031, 2927, 2868, 1497, 1454, 1406, 1365, 1208, 1096, 1056, 737, 699; MS (m/z): $222(\mathrm{M})^{+}, 207,191,181,161,143,131,116,101,91,83$, 65, 55, 43; HRMS: calcd. for (M-31) ${ }^{+} \mathrm{C}_{12} \mathrm{H}_{15} \mathrm{O}_{2}$ : 191.1071; found: 191.1064 .

\{(2R)-2-[2-(Benzyloxy)-ethyl]-tetrahydrofuran-2-yl\}-methanol 3e. Synthesis in $0.49 \mathrm{mmol}$ scale yielded THF-derivative as a colorless liquid (76 mg, 70\%). ${ }^{1} \mathrm{H}$ NMR (400 $\left.\mathrm{MHz}, \mathrm{CDCl}_{3}\right): \delta$ 1.74 (m, 1H, H-3), 1.89 (m, 5H, H-3, H-4, Et CH 2 ), 2.71 (bs, 1H, OH), 3.45 (m, 2H, $\mathrm{CH}_{2} \mathrm{OH}$ ), $3.57\left(\mathrm{~m}, 1 \mathrm{H}, \mathrm{CH}_{2} \mathrm{O}\right), 3.65\left(\mathrm{~m}, 1 \mathrm{H}, \mathrm{CH}_{2} \mathrm{O}\right), 3.82(\mathrm{~m}, 2 \mathrm{H}, \mathrm{H}-5), 4.51\left(\mathrm{~m}, 2 \mathrm{H}, \mathrm{Bn} \mathrm{CH}_{2}\right), 7.32(\mathrm{~m}$, $5 \mathrm{H}, \mathrm{Bn} o, p, m) ;{ }^{13} \mathrm{C} \mathrm{NMR}\left(100 \mathrm{MHz}, \mathrm{CDCl}_{3}\right): 26.11(\mathrm{C}-5), 33.15(\mathrm{C}-3), 36.86\left(\mathrm{Et} \mathrm{CH}_{2}\right), 66.86$ (Et $\left.\mathrm{CH}_{2} \mathrm{O}\right), 67.01(\mathrm{C}-5), 67.88\left(\mathrm{CH}_{2} \mathrm{OH}\right), 73.33\left(\mathrm{Bn} \mathrm{CH}_{2}\right), 84.35(\mathrm{C}-2), 127.65$ (p-Bn), 127.76 $(o-B n), 128.46(m-B n), 137.91(s-B n)$; IR (neat): 3444, 3088, 3063, 3030, 2947, 2868, 1496, 1454, 1366, 1308, 1206, 1098, 1053, 926, 738, 698; MS (m/z): 205 (M-31) $, 187,169,159,143$, 129, 113, 99, 91 (base), 81, 65; HRMS: calcd. for (M-31) ${ }^{+} \mathrm{C}_{13} \mathrm{H}_{17} \mathrm{O}_{2}$ : 205.1227; found: 205.1226; anal. calcd. for $\mathrm{C}_{14} \mathrm{H}_{20} \mathrm{O}_{3}$ : C, 71.16; H, 8.53; found: C, 70.89; H, 8.60.

(5R)-1,7-Dioxaspiro[4.4]nonane-2,6-dione 2g. Spirodilactone $2 \mathrm{~g}$ was synthesized with slight modification to previously reported method: To the solution of $\mathrm{Ti}(\mathrm{O} i \mathrm{Pr})_{4}(7.1 \mathrm{~mL}, 23.2 \mathrm{mmol})$ and (+)-DET (5.0 mL, $29.0 \mathrm{mmol})$ in DCM $(180 \mathrm{~mL})$ at -20 ${ }^{\circ} \mathrm{C}$ 2-hydroxy-3-(2hydroxyethyl)cyclopent-2-en-1-one $(3.3 \mathrm{~g}, 23.2 \mathrm{mmol})$ was added carefully. After stirring for 0.5 h $t$-BuOOH $(9.4 \mathrm{~mL}, 58.0 \mathrm{mmol})$ was added dropwise over $20 \mathrm{~min}$. The resulting mixture was kept at $-20{ }^{\circ} \mathrm{C}$ for $68 \mathrm{~h}$. The reaction was quenched with water $(145 \mathrm{~mL})$, then the mixture was stirred for $1 \mathrm{~h}$ and $10 \mathrm{~N} \mathrm{NaOH}$ solution $(29 \mathrm{~mL})$ was added. After stirring for $1 \mathrm{~h}$ the layers were separated and water phase was treated with $5.5 \mathrm{~N} \mathrm{HCl}$ solution $(110 \mathrm{~mL})$, then the water phase was extracted with DCM $(10 \times 100 \mathrm{~mL})$. The extracts were dried over $\mathrm{MgSO}_{4}$, filtered and the solvents evaporated to give $3.37 \mathrm{~g}$ of crude as yellow crystals, which upon crystallization from EtOAc/Et $\mathrm{E}_{2} \mathrm{O}$ mixture (1:4) gave spirodilactone $\mathbf{2 g}$ as white solids (2.75 g, $\left.17.6 \mathrm{mmol}, 75 \%\right)$, which physical and spectroscopic properties were in accordance with data given in literature ${ }^{42}$.

(R)-1,7-dioxaspiro[4,4]nonane 3g. Spirodilactone $\mathbf{2 g}$ (786 $\mathrm{mg}, 5.04 \mathrm{mmol})$ was dissolved in DCM (200 mL, solution was $0.025 \mathrm{M}$ in substrate, dried over $4 \AA \mathrm{MS}$, amylenes as stabilizers) under Ar atmosphere. Resulting solution was cooled to $-78^{\circ} \mathrm{C}$ and then DIBALH (7.0 mL, 10.6 mmol, 1.5M in toluene) was added dropwise. After $2 \mathrm{~h}$ stirring, $\mathrm{MeOH}$ (40 mL, dried over $3 \AA$ MS) and $\mathrm{BF}_{3} \cdot \mathrm{Et}_{2} \mathrm{O}(1.48 \mathrm{~mL}, 12.0 \mathrm{mmol})$ were added sequentially and the reaction mixture was kept at $-78^{\circ} \mathrm{C}$ for $14 \mathrm{~h}$ and then stirred $2 \mathrm{~h}$ at $23{ }^{\circ} \mathrm{C}$. The reaction was quenched with aq $\mathrm{NaHCO}_{3}$ $(30 \mathrm{~mL}, 10 \%)$ at $+4{ }^{\circ} \mathrm{C}$ and agitated for $1 \mathrm{~h}$, after which the layers were separated and the aqueous phase was extracted with DCM $(6 \times 50 \mathrm{~mL})$. Organic phase was dried over $\mathrm{MgSO}_{4}$, filtered and the solvents were evaporated to yield methylacetal 13 (565 mg, $3.0 \mathrm{mmol}, 60 \%)$ as yellow viscous oil, which was used in the next synthetic step without further purification. The crude and $\mathrm{Et}_{3} \mathrm{SiH}(2.9 \mathrm{~mL}, 18.0 \mathrm{mmol})$ were dissolved in DCM (60 mL, 0.05M in substrate), 
then the resulting solution was cooled to $-78{ }^{\circ} \mathrm{C}$ and $\mathrm{BF}_{3} \cdot \mathrm{Et}_{2} \mathrm{O}(750 \mathrm{uL}, 6.01 \mathrm{mmol})$ was added. The thus obtained reaction mixture was stirred for $3 \mathrm{~h}$ at $-78{ }^{\circ} \mathrm{C}$ and then the temperature was slowly allowed to reach $23{ }^{\circ} \mathrm{C}(4 \mathrm{~h})$. The reaction was quenched by adding aq $\mathrm{NaHCO}_{3}(5.0 \mathrm{~mL}$, $10 \%)$. The layers were separated and the water phase was extracted with DCM $(4 \times 30 \mathrm{~mL})$. The organics were dried over $\mathrm{MgSO}_{4}$, filtered and the solvents evaporated. Purification of the crude by flash chromatography $\left(\mathrm{SiO}_{2}\right.$, petroleum ether /acetone, 20:1 to 16:1) gave the title compound (123 mg, $0.96 \mathrm{mmol}, 32 \%)$ as light yellow oil. Caution! The volatility of title compound is of concern! $[\alpha]_{\mathrm{D}}{ }^{21}=-2.4\left(c 12.4, \mathrm{CHCl}_{3}\right) ;{ }^{1} \mathrm{H}$ NMR $\left(400 \mathrm{MHz}, \mathrm{CDCl}_{3}\right): \delta 1.85-1.92(\mathrm{~m}, 1 \mathrm{H}, \mathrm{H}-9)$, $1.89-2.01$ (m, 2H, H-3), $1.90-1.97$ (m, 2H, H-4), $2.02-2.10$ (m, 1H, H-9), 3.59 (d, J=9.1 Hz, 1H, H-6), 3.77 (d, $J=9.1 \mathrm{~Hz}, 1 \mathrm{H}, \mathrm{H}-6), 3.78-3.88$ (m, 2H, H-2), $3.87-3.97$ (m, 2H, H-8) and ${ }^{13} \mathrm{C}\left(100 \mathrm{MHz}, \mathrm{CDCl}_{3}\right): \delta 25.97(\mathrm{C}-3), 33.29(\mathrm{C}-4), 38.51(\mathrm{C}-9), 67.37$ (C-2), 67.99 (C-8), 76.90 (C-6), 89.16 (C-5); IR ( $\left.\mathrm{CHCl}_{3}\right): 2971,2870,1461,1059,911 ; \mathrm{MS}(\mathrm{m} / \mathrm{z}): 128,98,83,70,56$ (base), 42, 27; HRMS calcd. for (M) ${ }^{+} \mathrm{C}_{7} \mathrm{H}_{12} \mathrm{O}_{2}$ : 128.0836; found 128.0827 .

(3aR,7aR)-Tetrahydro-4H-furo[2,3-b]pyran-3a(7aH)-ol 12. Spirodilactone $2 \mathrm{~g}(156 \mathrm{mg}, 1.0$ $\mathrm{mmol}$ ) was dissolved in dry DCM ( $40 \mathrm{~mL}, 0.025 \mathrm{M}$ in substrate) under Ar atmosphere. Then, to the obtained solution TMSCl $(1.70 \mathrm{~mL}, 9.0 \mathrm{mmol})$ was added and the mixture was cooled to -78 ${ }^{\circ} \mathrm{C}$ after which DIBALH $(1.15 \mathrm{~mL}, 2.5 \mathrm{mmol})$ was added dropwise. Reaction mixture was stirred at $-78{ }^{\circ} \mathrm{C}$ for $2 \mathrm{~h}$ and carefully quenched with aq $\mathrm{NaHCO}_{3}(10 \%, 0.46 \mathrm{~mL})$ and the temperature rised slowly to $23{ }^{\circ} \mathrm{C}$. $\mathrm{Na}_{2} \mathrm{SO}_{4}(2.28 \mathrm{~g})$ was added and the stirring was continued for further $2 \mathrm{~h}$. The solids were filtered off and washed with EtOAc $(3 \times 10 \mathrm{~mL})$. Combined organics were concentrated and purified by flash chromatography $\left(\mathrm{SiO}_{2}\right.$, PE/acetone 10:1 to 3:1) to yield title compound as clear liquid (65 mg, $0.45 \mathrm{mmol}, 45 \%)$. $[\alpha]_{\mathrm{D}}{ }^{21}=-7.8\left(c 9.1, \mathrm{CHCl}_{3}\right) ;{ }^{1} \mathrm{H}$ NMR $(400$ $\left.\mathrm{MHz}, \mathrm{CDCl}_{3}\right): \delta 1.56(\mathrm{~m}, 1 \mathrm{H}, \mathrm{H}-4 \mathrm{e}), 1.64(\mathrm{~m}, 1 \mathrm{H}, \mathrm{H}-4 \mathrm{a}), 1.71(\mathrm{~m}, 1 \mathrm{H}, \mathrm{H}-7), 1.76(\mathrm{~m}, 1 \mathrm{H}, \mathrm{H}-5 \mathrm{a})$, 2.13 (m, 1H, H-5e), 2.24 (m, 1H, H-7), 2.86 (bs, 1H, OH), 3.39 (m, 1H, H-3a), 3.83 (m, 1H, H3e), 4.10 (m, 2H, H-8), 4.61 (s, 1H, H-1) and ${ }^{13} \mathrm{C} \mathrm{NMR} \mathrm{(100} \mathrm{MHz,} \mathrm{CDCl}_{3}$ ): 23.02 (C-4), 31.61 (C-5), 33.04 (C-7), 64.29 (C-3), 67.21 (C-8), 77.15 (C-6), 105.03 (C-1); IR (neat): 3428, 2948, 2899, 2862, 1447, 1252, 1213, 1128, 1100, 1070, 1034, 991, 966, 932, 726, 601, 588; MS (m/z): $144(\mathrm{M})^{+}, 126,116,98,97,70$ (base), 56; HRMS: calcd. for $(\mathrm{M})^{+} \mathrm{C}_{7} \mathrm{H}_{12} \mathrm{O}_{3}: 144.0785$; found: 144.0783 .

\section{Acknowledgements}

We are grateful to the Estonian Ministry of Education and Research (Grant No: 0142725s06), the Estonian Science Foundation (Grant No: 5628 and 7114), EU European Regional Development Fund 3.2.0101.08-0017, and the Competence Centre for Cancer Research for financially supporting to carrying out of this project. 


\section{References and Notes}

1. Che, Y.; Gloer, J. B.; Scott, J. A.; Malloch, D. Tetrahedron Lett. 2004, 45, 6891.

2. Bermejo, A.; Figad re, B.; Zafra-Polo, M.; Barrachina, I.; Estornell, E.; Cortes, D. Nat. Prod. Rep. 2005, 22, 269.

3. Kladi, M.; Vagias, C.; Papazafiri, P.; Brogi, S.; Tafi, A.; Roussis, V. J. Nat. Prod. 2009, 72, 190.

4. Yasumoto, T.; Muratat, M. Chem. Rev. 1993, 93, 1897.

5. Wong, H. N. C. Eur. J. Org. Chem. 1999, 1757.

6. Li, Y.; Zhou, F.; Forsyth, C. J. Angew. Chem., Int. Ed. 2007, 46, 279.

7. Wu, J.; Li, N.; Hasegawa, T.; Sakai, J.; Kakuta, S.; Tang, W.; Oka, S.; Kiuchi, M.; Ogura, H.; Kataoka, T.; Tomida, A.; Tsuruo, T.; Ando, M. J. Nat. Prod. 2005, 68, 1656.

8. Houlihan, W. J.; Lee, M. L.; Munder, P. G.; Nemecek, G. M.; Handley, D.; Winslow, C. M.; Happy, J.; Jaeggi, C. Lipids 1987, 22, 884.

9. Doherty, G. A.; Yang, G. X.; Borges, E.; Chang, L. L.; Maccoss, M.; Tong, S.; Kidambi, U.; Egger, L. A.; Mccauley, E.; Riper, G. V.; Mumford, R. A.; Schmidt, J. A.; Hagmann, W. K. Bioorg. Med. Chem. Lett. 2002, 12, 1501.

10. Khandelwal, A.; Narayanan, R.; Gopalakrishnan, B. Bioorg. Med. Chem. 2003, 11, 4235.

11. Lee, C.; Jiang, L.; Hang, H.; Hon, P.; He, Y.; Wong, H. N. C. Br. J. Pharmacol. 1991, 103, 1719.

12. Tasdemir, D.; Wright, A. D.; Sticher, O.; Çalis, I.; Linden, A. J. Nat. Prod. 1995, 58, 1543.

13. Midland, S. L.; Keen, N. T.; Sims, J. J.; Midland, M. M.; Stayton, M. M.; Burton, V.; Smith, M. J.; Mazzola, E. P.; Graham, K. J.; Clardy, J. J. Org. Chem. 1993, 58, 2940.

14. Babu, B. R.; Keinicke, L.; Petersen, M.; Nielsen, C.; Wengel, J. Org. Biomol. Chem. 2003, 1, 3514.

15. Cipolla, L.; Forni, E.; Jimenez-Barbero, J.; Nicotra, F. Chem. Eur. J. 2002, 3976.

16. Wolfe, J. P.; Hay, M. B. Tetrahedron 2007, 63, 261.

17. Shin, C.; Oh, Y.; Cha, J. H.; Pae, A. N.; Choo, H.; Cho, Y. S. Tetrahedron 2007, 63, 2182.

18. DeAngelis, A.; Taylor, M. T.; Fox, J. M. J. Am. Chem. Soc. 2009, 131, 1101.

19. Mitchell, T. A.; Zhao, C.; Romo, D. Angew. Chem., Int. Ed. 2008, 47, 5026.

20. Sim, J. Y.; Hwang, G.; Kim, K. H.; Ko, E. M.; Ryu, D. H. Chem. Commun. 2007, 5064.

21. Masuda, T.; Osako, K.; Shimizu, T.; Nakata, T. Org. Lett. 1999, 1, 941.

22. Maezaki, N.; Sawamoto, H.; Suzuki, T.; Yoshigami, R.; Tanaka, T. J. Org. Chem. 2004, 69, 8387.

23. Donohoe, T. J.; Butterworth, S. Angew. Chem., Int. Ed. 2005, 44, 4766.

24. Prasad, K.; Estermann, H.; Underwood, R. L.; Chen, C.; Kucerovy, A.; Repic, O. J. Org. Chem. 1995, 60, 7693.

25. Alonso, F.; Meléndez, J.; Yus, M. Tetrahedron Lett. 2004, 45, 1717.

26. Donohoe, T. J.; Fisher, J. W.; Edwards, P. J. Org. Lett. 2004, 6, 465.

27. Marco-Contelles, J.; Dominguez, L.; Anjum, S.; Ballesteros, P.; Soriano, E.; Postel, D. 
Tetrahedron: Asymmetry 2003, 14, 2865.

28. Paju, A.; Kanger, T.; Pehk, T.; Lindmaa, R.; Müürisepp, A.; Lopp, M. Tetrahedron: Asymmetry 2003, 14, 1565.

29. Jõgi, A.; Paju, A.; Pehk, T.; Kailas, T.; Müürisepp, A.; Kanger, T.; Lopp, M. Synthesis 2006, 2006, 3031.

30. Paju, A.; Laos, M.; Jõgi, A.; Päri, M.; Jäälaid, R.; Pehk, T.; Kanger, T.; Lopp, M.

Tetrahedron Lett. 2006, 47, 4491.

31. Paju, A.; Kanger, T.; Pehk, T.; Eek, M.; Lopp, M. Tetrahedron 2004, 60, 9081.

32. Jõgi, A.; Ilves, M.; Paju, A.; Kailas, T.; Müürisepp, A.; Lopp, M. Tetrahedron: Asymmetry 2008, 19, 628.

33. Jõgi, A.; Paju, A.; Pehk, T.; Kailas, T.; Müürisepp, A.; Lopp, M. Tetrahedron 2009, 65, 2959.

34. Verma, P.; Singh, S.; Dikshit, D. K.; Ray, S. Synthesis 1988, 1988, 68.

35. Ravid, U.; Silverstein, R. M.; Smith, L. R. Tetrahedron 1978, 34, 1449.

36. Pettit, G. R.; Kasturi, T. R. J. Org. Chem. 1961, 26, 4557.

37. Kraus, G. A.; Frazier, K. A.; Roth, B. D.; Neuenschwander, K. J. Org. Chem. 1981, 46, 2417.

38. Mao, Z.; Gregg, B. T.; Cutler, A. R. J. Am. Chem. Soc. 1995, 117, 10139.

39. Hansen, M. C.; Verdaguer, X.; Buchwald, S. L. J. Org. Chem. 1998, 63, 2360.

40. Yato, M.; Homma, K.; Ishida, A. Tetrahedron 2001, 57, 5353.

41. Matsubara, K.; Iura, T.; Maki, T.; Nagashima, H. J. Org. Chem. 2002, 67, 4985.

42. Paju, A.; Kanger, T.; Niitsoo, O.; Pehk, T.; Müürisepp, A.; Lopp, M. Tetrahedron: Asymmetry 2003, 14, 2393.

43. The number of lactol carbons in the range of 94 to $110 \mathrm{ppm}$ exceeds that of possible when only spirocyclic acetals 9 are counted (12 vs 8), thus indicating a presence of other lactols. Most probably accountable for lactolalcohols $\mathbf{1 0}$ and $\mathbf{1 1}$ as depicted on the scheme 5. Also the number of quaternary and oxygen-linked carbons support the hypothesis of presence of overreduction products in the crude product.

44. Paquette, L. A.; Seekamp, C. K.; Kahane, A. L. J. Org. Chem. 2003, 68, 8614.

45. Chandrasekhar, S.; Tiwari, B.; Prakash, S. J. Arkivoc 2006, 2006, 155.

46. Brueckner, C.; Holzinger, H.; Reissig, H. U. J. Org. Chem. 1988, 53, 2450.

47. According to Kraus' work (see ref 37) the TBDMS group should be stable under the reaction conditions described. 